\title{
Modified Harmony Search Algorithm for Solving the Four-Color Mapping Problem
}

\author{
Bnar Faisal A. Daham \\ Software Engineering Dept.
}

\author{
Mohammed N. Mohammed \\ Surveying Engineering Dept.
}

Kanar Sh. Mohammed
Software Engineering Dept.

College of Engineering, University of Salahaddin, Erbil - Iraq

\begin{abstract}
The Four-Color Mapping Problem has been solved using different optimization algorithms. Harmony Search (HS) is one of those algorithms, which is based on the imitation of the behavior of musicians when composing their music. The HS algorithm can be summarized in three steps... initialization, improvisation, and selection.

We introduced in this paper an approach to enhance the performance of HS algorithm, in solving the Four-Color Mapping Problem. A modification has been applied to the initialization section of the HS algorithm, which affects the improvisation process, resulting in a boost in the performance of the improvisation process, and consequently, reducing the time and number of cycles taken to solve the Four-Color Mapping Problem compared to the HS algorithm. In this paper, tests have been carried out on maps with different numbers of regions, using both HS and Modified Harmony Search (MHS) algorithms. The obtained results of the MHS algorithm are better than those of the original HS one.
\end{abstract}

\section{Keywords}

Optimization, Four-Color Mapping Problem, Harmony Search Algorithm.

\section{INTRODUCTION}

Four Color Mapping is one of the combinatorial optimization problems that was first conjectured in 1852 by Francis Guthrie [1][2]. The 4-Color Mapping can be said that the regions of any simple planar map can be colored with only four colors, in such a way that any two adjacent regions have different colors [3][4][5]. Two regions are said to be adjacent if and only if a common boundary is shared, not just a common point [4][6]. Figure 1 illustrates the adjacency of regions in the given sample. Regions A and B have a common boundary, same goes with regions $\mathrm{C}$ and $\mathrm{D}$. But regions $\mathrm{A}$ and $\mathrm{D}$ only share a common point, so do regions $\mathrm{B}$ and $\mathrm{C}$. Therefore, by definition, regions $\mathrm{A}$ and $\mathrm{B}$ are adjacent, as well as regions $\mathrm{C}$ and $\mathrm{D}$, but not regions $\mathrm{A}$ and $\mathrm{D}$, and regions $\mathrm{B}$ and $\mathrm{C}$ [6]. Figure 2 illustrates 29 regions have been colored with four colors and no adjacent regions have the same color.

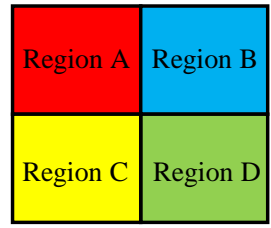

Figure 1: Adjacency of regions

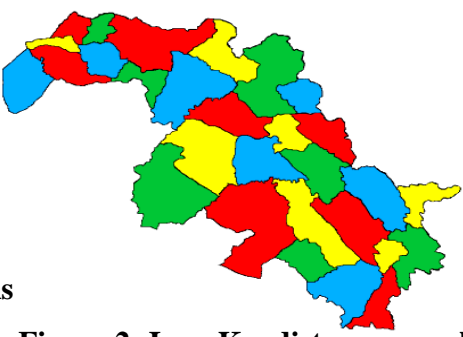

Figure 2: Iraq-Kurdistan map colored using only four colors
As the HS algorithm randomly generates harmonies or candidate solutions, a modification is applied to it in this paper by letting it generate the harmonies, then randomly assigning and fixing - but separately from HS improvisation process - the four different colors to the first four regions. Thus saving time and cycles specifically in solving the Four-Color Mapping Problem.

In the rest of the paper; HS algorithm is overviewed in Section 2. Section 3 describes MHS algorithm, and solving the FourColor Mapping Problem using MHS algorithm is described in section 4. In section 5, experimental results are presented. Finally, some concluding remarks are presented in Section 6.

\section{HARMONY SEARCH ALGORITHM}

Computer scientists have found a significant relationship between music and the process of looking for an optimal solution. This interesting connection led to the creation of the HS algorithm. It is a new kind of meta-heuristic algorithm mimicking a musicians' approach to finding harmony while playing music [7]. When musicians try to create music, they may use one or a combination of the three possible methods for musical improvisation which are as follows: (1) playing the original piece, (2) playing in a way similar to the original piece, and (3) creating a piece through random notes [6][8].

Zong Woo Geem et al. [7][9][10], noticed the similarity of this behavior in achieving the optimal solution to a problem. In 2001, he proposed three methods corresponding to the three techniques namely the use of (1) random selection, (2) memory consideration, (3) and pitch adjustment. These became the elements of the newly developed meta-heuristic optimization algorithm called the HS algorithm [6][7]. Figure 3 illustrates the basic Harmony Search Algorithm [6][7][8].

\footnotetext{
Begin

Define Objective function $\mathrm{f}(\mathrm{x}), \mathrm{x}=(\mathrm{x} 1, \mathrm{x} 2, \ldots, \mathrm{xd})^{\mathrm{T}}$

Define harmony memory accepting rate $\left(\mathrm{r}_{\text {accept }}\right)$

Define pitch adjusting rate $\left(\mathrm{r}_{\mathrm{pa}}\right)$, pitch limits and bandwidth

Generate Harmony Memory with random

harmonies

While $(\mathrm{t}<\max$ number of iterations (cycles))

While ( $\mathrm{t}<\max$ number of variables)

If $\left(\right.$ rand $<r_{\text {accept }}$ ), choose a value of var $\mathrm{i}$

If $\left(\right.$ rand $<r_{p a}$ ), adjust the value

End if

Else choose a random value

End if

End while

Accept a new harmonics (solutions) if better

End while

Choose the best solution

End
}

Figure 3: Basic Harmony Search algorithm 
Mathematically, the design of a basic HS algorithm is generally based on the following steps [10][11][12]

Step 1. Initialize the problem and algorithm parameters.

Step 2. Initialize the Harmony Memory (HM).

Step 3. Improvise a New Harmony memory.

Step 4. Update the Harmony memory.

Step 5. Check the stopping criterion.

The capability of Harmony Search Algorithm in solving problems has been proven effective in various studies. In this study, the researchers aim to determine the feasibility of the said algorithm and its modified in solving Four-Color Mapping problem [6].

\section{MODIFIED HARMONY SEARCH (MHS) ALGORITHM}

In this paper an approach is introduced to enhance the performance of HS algorithm specifically for solving the FourColor Mapping Problem. A modification has been applied to the initialization section of the HS algorithm. That is by excluding the first four elements of each vector (candidate solution) from any computations of improvisation process of HS algorithm.

After generating the harmonies, the first four elements in each of them will be assigned randomly four different values. The assignment should involve the available different values to achieve and guarantee the condition of Four-Color Mapping Problem. Also the assigned values will be fixed per a solution because they are not included in the improvisation process (Random Selection, Memory Consideration, and Pitch Adjustment). In other words, the improvisation process will treat $(n-4)$ elements, where $n$ is the number of the regions in the processed map. As a result, the path to solve the Four-Color Mapping Problem will be shortened compared to the original HS algorithm through reducing the time and number of cycles taken to reach the optimal solution. Figure 4 and 5 illustrates the MHS algorithm and the design of general steps of the MHS algorithm respectively.

\section{Begin}

Define Objective function $\mathrm{f}(\mathrm{x}), \mathrm{x}=(\mathrm{x} 1, \mathrm{x} 2, \ldots, \mathrm{xd})^{\mathrm{T}}$

Define harmony memory accepting rate $\left(\mathrm{r}_{\text {accept }}\right)$

Define pitch adjusting rate $\left(\mathrm{r}_{\mathrm{pa}}\right)$, pitch limits and bandwidth

Generate Modified Harmony Memory with random harmonies and assign randomly the four different colors to the first four regions for each pattern in the MHM as many as HMS

While $(\mathrm{t}<\max$ number of iterations (cycles))

While ( $\mathrm{t}<\max$ number of variables)

If (rand $\left.<r_{\text {accept }}\right)$, choose a value of var $i$

If $\left(\right.$ rand $<r_{\mathrm{pa}}$ ), adjust the value

End if

Else choose a random value

End if

\section{End while}

Accept a new harmonics (solutions) if better

End while

Choose the best solution

End

Figure 4: Modified Harmony Search algorithm

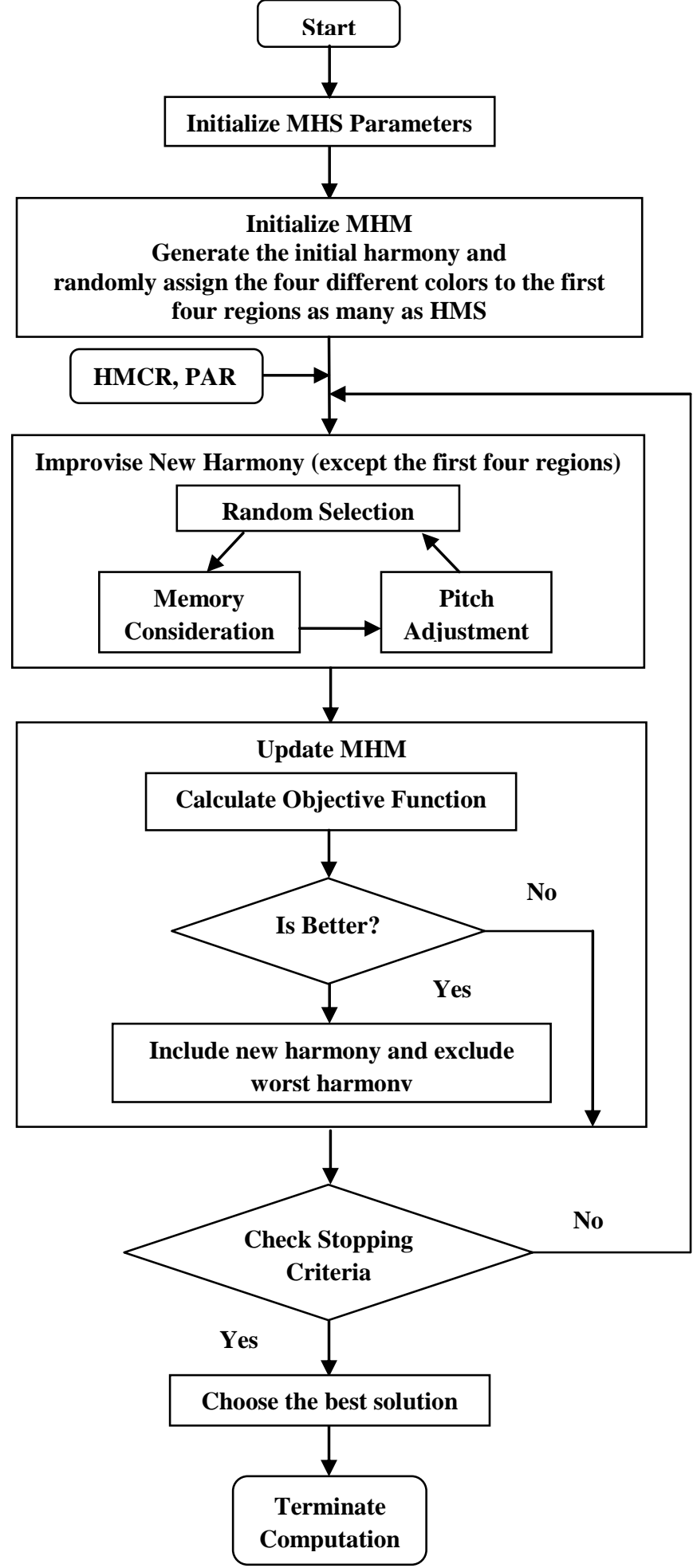

Figure 5: Steps of Modified Harmony Search algorithm 


\section{SOLVING THE FOUR-COLOR MAPPING PROBLEM USING MHS ALGORITHM}

In this section, we will discuss the approach that the researchers took in achieving a four-colored map solution. Figure 6 illustrates the general flow of the proposed approach.

Each candidate solution is generated as a vector whose variables correspond to the regions of the map. Vectors are arrays of integer variables that serve as the solution to the problem. Regions to be colored take values 1, 2, 3, or 4 representing the four colors where each number stands for a color in the solution such as $1=$ red, $2=$ green, $3=$ blue and $4=$ yellow. The size of the solution is equivalent to the number of regions in the map. Figure 7 shows the representation of the solution. The general formula of each candidate solution is as follows:

$\mathbf{R}=\langle\mathbf{R} 1, \mathbf{R} 2, \mathbf{R 3}$

where $\mathbf{R}$ represents the regions having values $1,2,3$, or 4 and $\mathbf{n}$ is the number of regions in the map. The optimization process starts by generating random solutions in the harmony memory. Equation (1) illustrates the characteristics of each candidate solution in the memory. After initializing the harmony memory, it is followed by the improvisation process. Each possible solution is evaluated for its respective objective value. Then the optimization process ends if the process generates an optimal solution or when the maximum improvisation is reached. This is where a new harmony or a possible solution is generated through random selection, harmony memory consideration or pitch adjustment.

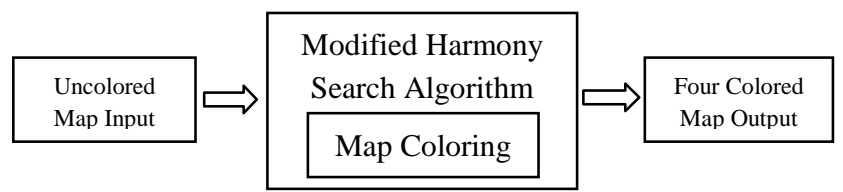

Figure 6: MHS block diagram for solving four-color mapping problem

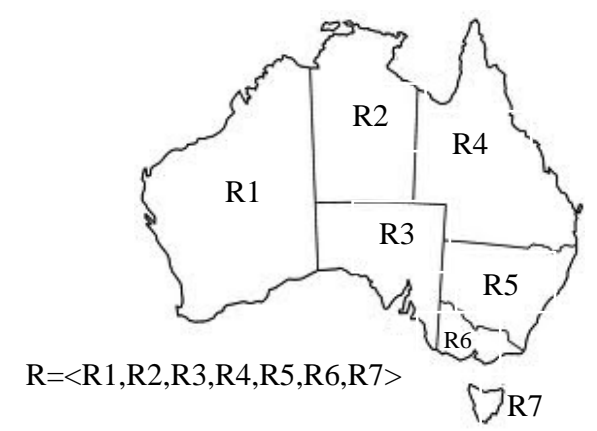

Figure 7: Vector representation for Australia map

The design of a basic MHS algorithm generally includes the following steps:

1. Initialize MHS algorithm Parameters: In order for the Harmony Search Algorithm to start, the following parameters must be set:

- Number of decision variables: Each harmony is composed of several decision variables.

- Number of cycles (iterations): One of the termination conditions of the optimization process.
- Harmony Memory Size: Refers to the number of solutions that will be stored in the harmony memory.

- Harmony Memory Consideration Rate: The rate at which the value of the decision variables from the harmony memory is picked as elements of the new harmony that will be created.

- Pitch Adjustment Rate: The probability that the decision variable picked from the harmony memory be altered by some certain amount.

Table 1 shows the standard parameter values used for the experiments.

Table 1: Standard MHS algorithm parameter values

\begin{tabular}{|c|c|c|c|c|c|}
\hline $\begin{array}{c}\text { Parameter } \\
\text { S }\end{array}$ & $\begin{array}{c}\text { No. of } \\
\text { Variable } \\
\text { s }\end{array}$ & Cycles & $\begin{array}{c}\text { HM } \\
\text { S }\end{array}$ & $\begin{array}{c}\text { HMC } \\
\mathbf{R}\end{array}$ & $\begin{array}{c}\text { PA } \\
\text { R }\end{array}$ \\
\hline Values & $\begin{array}{c}\text { No. of } \\
\text { Regions } \\
\text { in the } \\
\text { map }\end{array}$ & $\begin{array}{c}100000 \\
0\end{array}$ & 10 & 0.9 & 0.4 \\
\hline
\end{tabular}

\section{Initialize MHM}

In this step we generate the initial MHM normally then randomly assign the four different colors to the first four regions as many as HMS as shown in Figure 8.

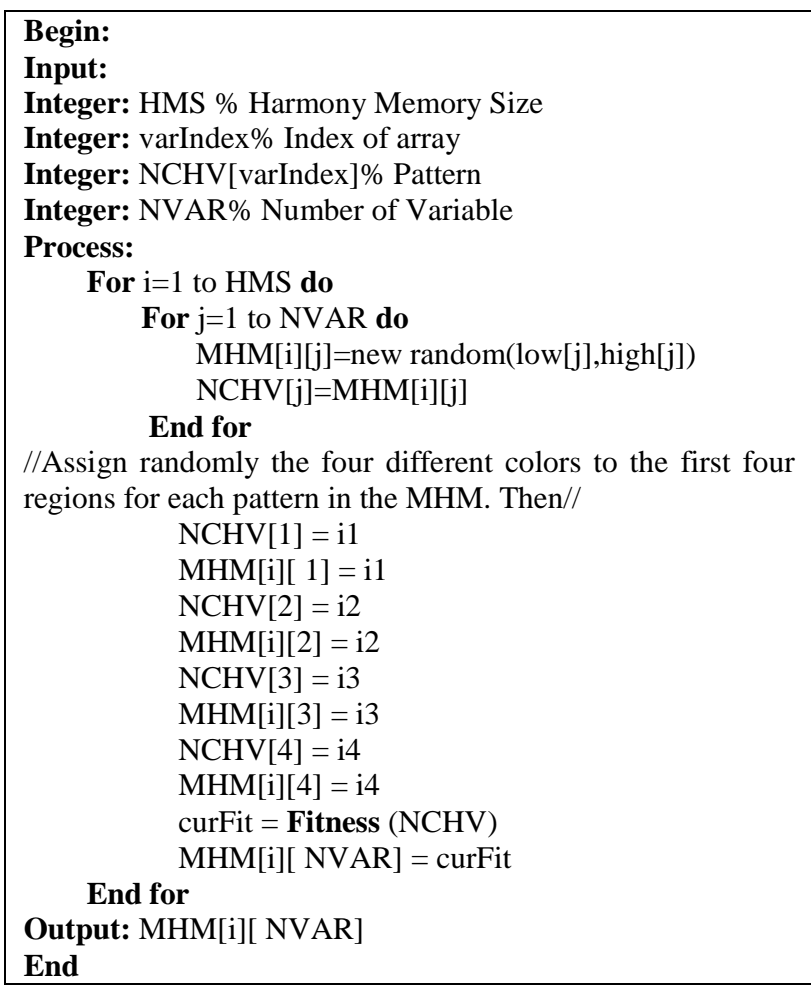

Figure 8: Modified Harmony Memory initialization

For example if we have a 7-region map the first four regions after harmonies generation will be $[1,2,3,4],[2,4,3,1],[3,2,4,1]$, $[1,3,2,4],[4,3,1,2],[2,1,3,4]$, and so on. This in turn eliminates any probability of having duplicated colors in those four regions like $[1,1,2,3],[1,2,1,1],[2,2,3,4],[3,3,3,3]$, and so like.

\section{Random Selection}

Random Selection is where a variable in the harmony takes a random value; in this problem it will select one random color 
from the available four options. To be noticed, in our case, random selection, memory consideration, and pitch adjustment will start from region five and upwards.

\section{Memory Consideration}

Values or colors in the harmony memory have a chance to be selected when performing memory consideration. So memory consideration for region five will be the available colors for region five in the modified harmony memory.

\section{Pitch Adjustment}

Selected value from the memory can be altered to obtain a variation through pitch adjustment. Figure 9 illustrates how pitch adjustment is done.

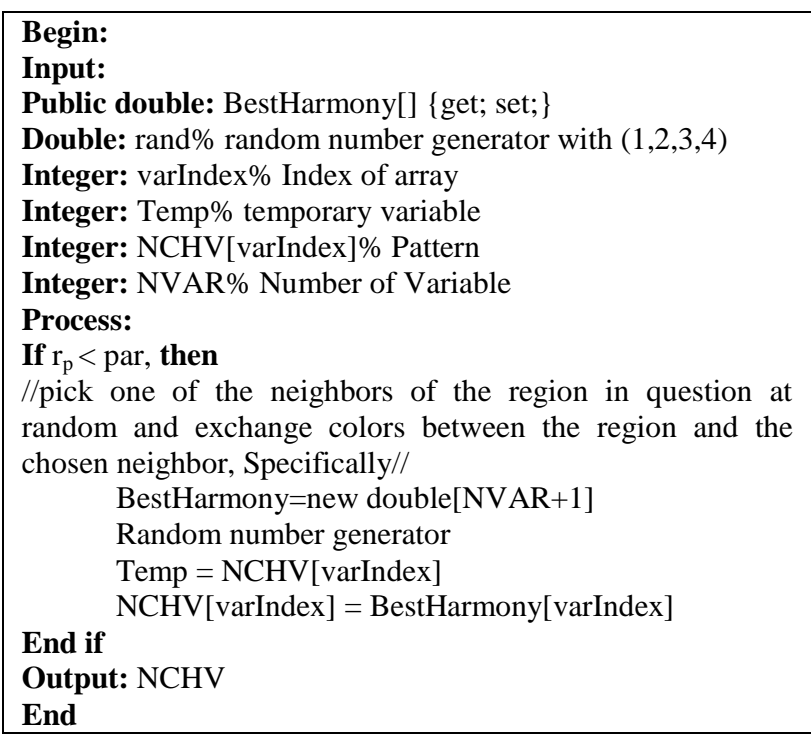

\section{Figure 9: Pitch Adjustment process}

\section{Objective (Fitness) Function}

The evaluation function will return a value that will represent the fitness of a harmony vector. The function aims to get a minimum result, wherein, the smaller the fitness value, the better the vector is. The value also represents the number of errors present in the solution. If the fitness value reaches zero, then that is the optimal solution and the optimization process will stop. Figure 10 illustrates how the objective function works.

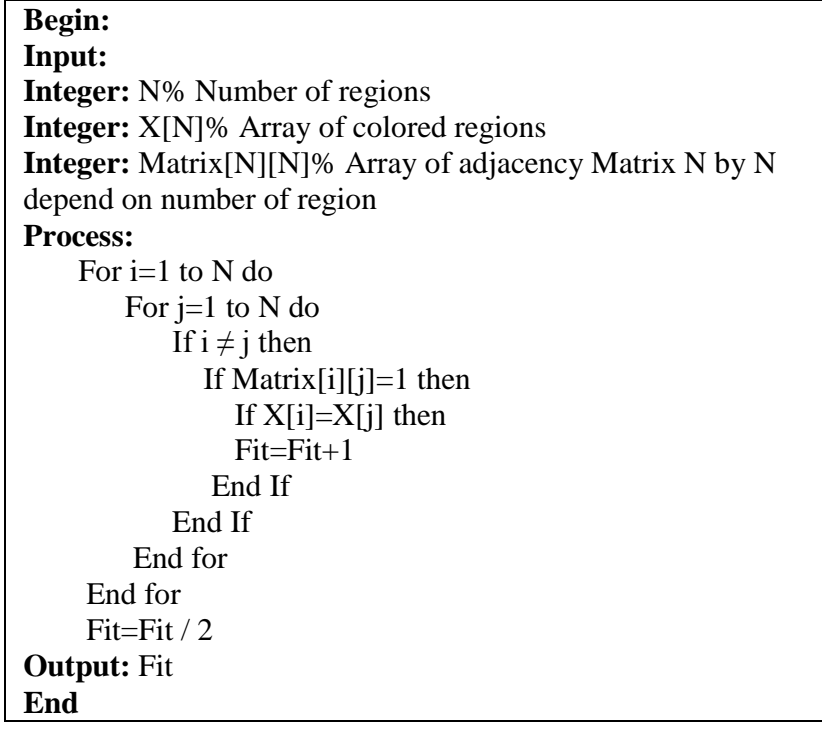

Figure 10: Objective function process
7. Illustrating the MHS Algorithm Process to Map Coloring Using the map in Figure 7, Initialize random harmony vectors that will represent the solution for the colored map using equation 1 . In this example, we set our harmony memory to two so that $\mathrm{HMS}=2, \mathrm{R}^{1}=[4,1,3,2,1,1,3]$ and $\mathrm{R}^{2}=[1,3,2,4,4,3,4]$. Then improvise a new harmony vector through Random Selection, Memory Consideration, and Pitch Adjustment. After that we evaluate the harmony using the objective function process in Figure 10. Only half of the matrix is evaluated since the matrix is symmetric. The smaller resulting value is the better result. $\mathrm{R}^{1}$ is evaluated as shown bellow. Using the same function, the result of $\mathrm{R}^{2}=1$ also.

$$
\begin{array}{r}
\mathrm{R}^{1}=0 \times 0+1 \times 0+1 \times 0+0 \times 0+0 \times 0+0 \times 0+0 \times 0 \\
0 \times 0+1 \times 0+1 \times 0+0 \times 0+0 \times 0+0 \times 0 \\
0 \times 0+1 \times 0+1 \times 0+1 \times 0+0 \times 0 \\
0 \times 0+1 \times 0+0 \times 0+0 \times 0 \\
0 \times 0+1 \times 1+0 \times 0 \\
0 \times 0+0 \times 0 \\
0 \times 0
\end{array}
$$

Both improvisation of a new harmony vector and evaluation of a new harmony are repeated until a series of finite number of improvisations is achieved. The best harmony in the harmony memory is selected to become the solution. The map will be colored using the corresponding colors of the values in the solution.

\section{TESTS AND RESULTS}

HS and MHS algorithms were implemented in Microsoft Visual C\# 2010 and run on a computer whose processor is $\operatorname{Intel}(\mathrm{R})$ Core $^{\mathrm{TM}} 2.50 \mathrm{GHz}$, with $6 \mathrm{~GB}$ main memory. The algorithms were applied on 7 regions Australia map, 10 regions Austria map, 18 regions Iraqi map, 29 regions Kurdistan-Iraq map, 32 regions China map, 48 regions USA map, and finally 50 regions Africa continent map.

The comparison between the original and modified HS algorithms will depend on the time and number of cycles required to find the optimal solutions for each vector of regions. Ten to fifteen runs for each instance have been achieved and the standard deviation of time and cycles required to reach the optimal solution have been reported in Table 2 .

Table 2: Standard deviation of elapsed time and number of cycles for HS and MHS algorithms for solving Four-Color Mapping problem

\begin{tabular}{|c|c|c|c|c|}
\hline \multirow{2}{*}{ Regions } & \multicolumn{2}{|c|}{ Time } & \multicolumn{2}{c|}{ Cycles } \\
\cline { 2 - 5 } & HS & MHS & HS & MHS \\
\hline 7 & 0.007656975 & 0.005310974 & 10.07223908 & 2.015495528 \\
\hline 10 & 0.006988317 & 0.006027705 & 99.88413287 & 39.54367812 \\
\hline 18 & 0.173869271 & 0.086737074 & 1939.580357 & 1036.398995 \\
\hline 29 & 0.889333565 & 0.316231162 & 7042.100332 & 2046.595126 \\
\hline 32 & 1.073332541 & 0.616941191 & 7576.397402 & 3331.733635 \\
\hline 48 & 6.400353566 & 6.022102219 & 21703.68652 & 21212.37458 \\
\hline 50 & 7.961991411 & 7.513218207 & 26454.82923 & 23563.50263 \\
\hline
\end{tabular}

Table 2 shows how the MHS algorithm has higher performance than the HS algorithm. Figures 11 and 12 are illustrating the difference between the performance of both MHS \& HS algorithms in reaching the optimal solution in terms of time and number of cycles respectively. 


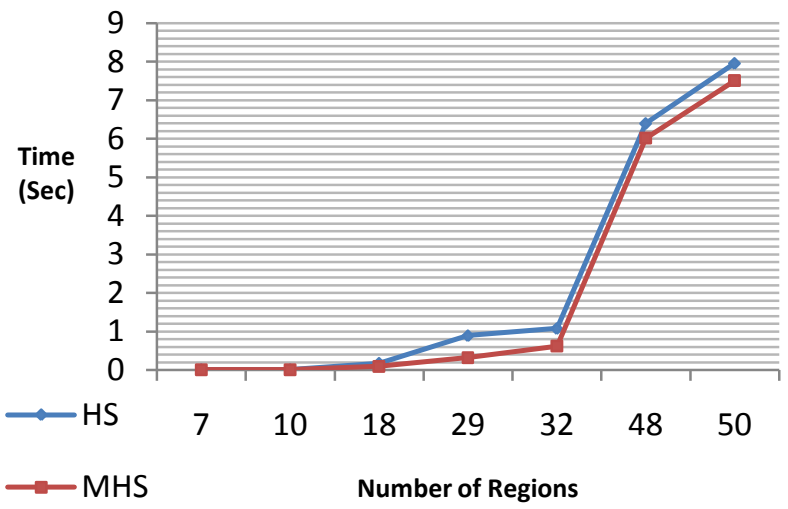

Figure 11: Difference in elapsed time between HS and MHS algorithms

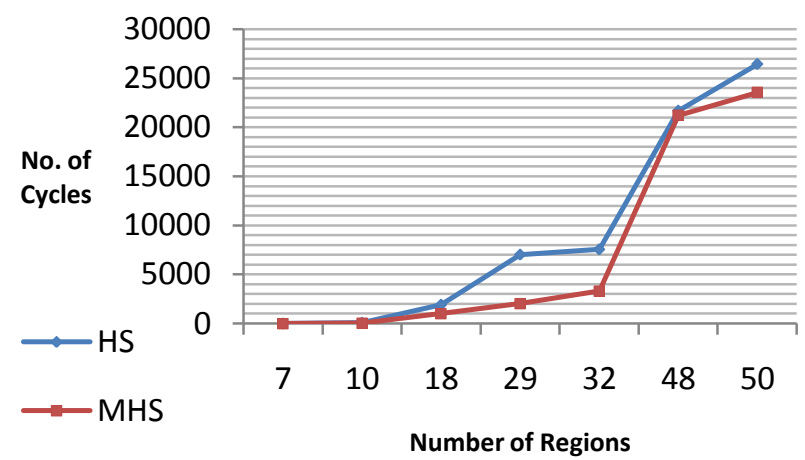

Figure 12: Difference in number of cycles between HS and MHS algorithms

\section{CONCLUSIONS}

The computational results illustrate that the MHS algorithm presents better performance than the original HS algorithm where the MHS reached the optimal solution faster than the original HS applying tests on maps with different number of regions. The results also indicate that the time and number of cycles increase as the size of the map increases. This is because it will be more difficult to find an optimal solution as the size of the map gets bigger.

For future work hybridization can be applied between HS and another optimization algorithm. One of the approaches is to use Ant or Bee colony algorithms in generating the harmonies (candidate solutions) instead of producing them randomly.

\section{REFERENCE}

[1] Robin Wilson, "Four Colours Suffice", Allen Penguin Press books, 2002.
[2] Mohammed S. Ibrahim, Ahmed T. Sadiq, and Ali M. Sagheer, "Hybrid Scatter Search Algorithm for 4Color Mapping Problem", the international Arab Conference on Information Technology ACIT 2012 Dec. 10-13, ISSN 1812-0857.

[3] Georges Gonthier, "Formal Proof-The FourColor Theorem", Notices of the AMS, Volume 55, Number 11, December 2008.

[4] Georges Gonthier, "A computer-checked proof of the Four Colour Theorem", Microsoft Research Cambridge, 2005.

[5] Andreea S. Calude, "The Journey of the Four Colour Theorem Through Time", Department of Mathematics, The University of Auckland, New Zealand, December 2000.

[6] Romie B. Horca and John Paul T. Yusiong, "Using Harmony Search Algorithm to Solve the N-Region Four Color Map Problem", Journal of Applied Computer Science \& Mathematics, no. 12 (36), Suceava, 2012.

[7] Victor M. Romero, Leonel L. Tomes, and John Paul T. Yusiong, "Tetris Agent Optimization Using Harmony Search Algorithm", International Journal of Computer Science Issues, Vol. 8, Issue 1, January 2011.

[8] Xin-She Yang, "Harmony Search as a Metaheuristic Algorithm", in: Music-Inspired Harmony Search Algorithm: Theory and Applications (Editor Z. W. Geem), Studies in Computational Intelligence, Springer Berlin, vol. 191, pp. 1-14, 2009.

[9] Kang Seok Lee and Zong Woo Geem, "A new metaheuristic algorithm for continuous engineering optimization: harmony search theory and practice", Elsevier B.V., 2004.

[10] Sachin A. Patil and D. A. Patel, "An Overview: Improved Harmony Search Algorithm and Its Applications in Mechanical Engineering", International Journal of Engineering Science and Innovative Technology (IJESIT) Volume 2, Issue 1, January 2013.

[11] Xiaobo Liu, Zhihua Cai, and Chao Yu, "A Hybrid Harmony Search Approach Based on Differential Evolution", Journal of Information \& Computational Science, Available at http://www.joics.com, Binary Information Press, October 2011.

[12] Parikshit Yadav, Rajesh Kumar, S.K. Panda, and C.S. Chang, "An Intelligent Tuned Harmony Search algorithm for optimisation", Department of Electrical and Computer Engineering, National University of Singapore, 2012. 